\title{
ON ÉTALE FUNDAMENTAL GROUPS OF FORMAL FIBRES OF $p$-ADIC CURVES
}

\author{
MOHAMED SAÏDI
}

\begin{abstract}
We investigate a certain class of (geometric) finite (Galois) coverings of formal fibres of $p$-adic curves and the corresponding quotient of the (geometric) étale fundamental group. A key result in our investigation is that these (Galois) coverings can be compactified to finite (Galois) coverings of proper $p$-adic curves. We also prove that the maximal prime-to- $p$ quotient of the geometric étale fundamental group of a (geometrically connected) formal fibre of a $p$-adic curve is (pro-)prime-to- $p$ free of finite computable rank.
\end{abstract}

\section{Contents}

$\S 0$. Introduction/Main Results

$\S 1$. Background

$\S 2$. Geometric Galois groups of formal boundaries of formal germs of $p$-adic formal curves

$\S 3$. Geometric fundamental groups of formal fibres of $p$-adic curves

$\S 0$. Introduction/Main Results. A classical result in the theory of étale fundamental groups is the description of the structure of the geometric étale fundamental group of an affine, smooth, and geometrically connected curve over a field of characteristic 0 (cf. [Grothendieck], Exposé XIII, Corollaire 2.12). In this paper we investigate the structure of a certain quotient of the geometric étale fundamental group of a formal fibre of a $p$-adic curve.

Let $R$ be a complete discrete valuation ring, $K=\operatorname{Fr}(R)$ its quotient field, and $k$ its residue field which we assume to be algebraically closed of characteristic $p \geq 0$. Let $X$ be a proper, flat, and normal formal $R$-curve whose special fibre $X_{k}$ is reduced and consists of $n \geq 1$ distinct irreducible components $\left\{P_{i}\right\}_{i=1}^{n}$ which intersect at a (closed) point $x \in X_{k}(k)$, and $x$ is the unique singular point of $X_{k}$. Write $\widetilde{P}_{i} \rightarrow P_{i}$ for the morphism of normalisation. We assume $\widetilde{P}_{i}=\mathbb{P}_{k}^{1}$ is a projective line, the morphism $\widetilde{P}_{i} \rightarrow P_{i}$ is a homeomorphism, and if $x_{i}$ is the (unique) pre-image of $x$ in $\widetilde{P}_{i}$ then $x_{i} \in \widetilde{P}_{i}(k)$ is the zero point of $\widetilde{P}_{i}$. In particular, the configuration of the irreducible components of $X_{k}$ is tree-like. The formal curve $X$ has a formal covering $X=B \cup D_{1} \cup \ldots \cup D_{n}$ where $B \subset X$ is a formal sub-scheme with

\footnotetext{
${ }^{1} 2010$ Mathematics Subject Classification. Primary 14H30; Secondary 11G20.

${ }^{2}$ Key words and phrases. Formal fibre, $p$-adic curves, Étale fundamental groups.
} 
special fibre $B_{k}=X_{k} \backslash\left\{\infty_{i}\right\}_{i=1}^{n}\left(\infty_{i}\right.$ is the image in $P_{i}$ of the infinity point of $\left.\widetilde{P}_{i}, 1 \leq i \leq n\right), D_{i}=\operatorname{Spf}\left\langle\frac{1}{T_{i}}\right\rangle$ is an $R$-formal closed unit disc with special fibre $D_{i, k}=P_{i} \backslash\{x\}$ and generic fibre $D_{i, K}=\operatorname{Sp} K\left\langle\frac{1}{T_{i}}\right\rangle$ which is a closed unit $K$ rigid disc centred at the point $\infty_{i} \in D_{i, K}(K)$ (which specialises in $\infty_{i} \in D_{i, k}$ ), $1 \leq i \leq n$. Write $\mathcal{F} \stackrel{\text { def }}{=} \mathcal{F}_{x}=\operatorname{Spf} \hat{\mathcal{O}}_{X, x}$ for the formal germ of $X$ at $x$ and $\mathcal{F}_{K} \stackrel{\text { def }}{=} \mathcal{F}_{x, K}=\operatorname{Spec}\left(\hat{\mathcal{O}}_{X, x} \otimes_{R} K\right)$ for the formal fibre of the generic fibre $X_{K}$ of the algebraisation of $X$ at $x$ (cf. 1.2 for more details, as well as Remark 3.1 which asserts that any formal germ of a formal $R$-curve at a closed point admits a compactification as above).

Let $S \subset \mathcal{F}_{K}$ be a (possibly empty) finite set of closed points. Write $\pi_{1}\left(\mathcal{F}_{K} \backslash S\right)^{\text {geo }}$ for the geometric étale fundamental group of $\mathcal{F}_{K} \backslash S$ (in the sense of Grothendieck, cf. 1.3 for more details), and consider the quotient $\pi_{1}\left(\mathcal{F}_{K} \backslash S\right)^{\text {geo }} \rightarrow \widehat{\pi}_{1}\left(\mathcal{F}_{K} \backslash S\right)^{\text {geo }}$ which classifies finite coverings $\mathcal{Y}^{\prime} \rightarrow \mathcal{F}^{\prime} \stackrel{\text { def }}{=} \mathcal{F} \times{ }_{R} R^{\prime}$, where $R^{\prime} / R$ is a finite extension, $\mathcal{Y}^{\prime}$ is normal and geometrically connected, which are étale above $\mathcal{F}_{K^{\prime}} \backslash S_{K^{\prime}}\left(K^{\prime} \stackrel{\text { def }}{=} \operatorname{Fr} R^{\prime}\right.$ and $\left.S_{K^{\prime}} \stackrel{\text { def }}{=} S \times_{K} K^{\prime}\right)$ and étale above the generic points of $\mathcal{F}_{k}$ (cf. loc. cit.). Similarly, write $\pi_{1}\left(X_{K} \backslash\left(S \cup\left\{\infty_{i}\right\}_{i=1}^{n}\right)\right)^{\text {geo }}$ for the geometric étale fundamental group of the affine curve $X_{K} \backslash\left(S \cup\left\{\infty_{i}\right\}_{i=1}^{n}\right)$ and consider the quotient $\pi_{1}\left(X_{K} \backslash\right.$ $\left.\left(S \cup\left\{\infty_{i}\right\}_{i=1}^{n}\right)\right)^{\text {geo }} \rightarrow \widehat{\pi}_{1}\left(X_{K} \backslash\left(S \cup\left\{\infty_{i}\right\}_{i=1}^{n}\right) ;\left\{\infty_{i}\right\}_{i=1}^{n}\right)^{\text {geo }}$ which classifies finite coverings $Y^{\prime} \rightarrow X_{R^{\prime}}$ which are étale above $X_{K^{\prime}} \backslash\left(S_{K^{\prime}} \cup\left\{\infty_{i}\right\}_{i=1}^{n}\right)$, possibly ramified above the points $\left\{\infty_{i}\right\}_{i=1}^{n}$ with ramification indices prime-to- $p$, and which are étale above the generic points of $X_{k}$ (here $R^{\prime}, K^{\prime}$ and $S_{K^{\prime}}$ are as above). We also write $\widehat{\pi}_{1}\left(X_{K} \backslash\left(S \cup\left\{\infty_{i}\right\}_{i=1}^{n}\right)\right)^{\text {geo }, p} \stackrel{\text { def }}{=} \widehat{\pi}_{1}\left(X_{K} \backslash\left(S \cup\left\{\infty_{i}\right\}_{i=1}^{n}\right) ;\left\{\infty_{i}\right\}_{i=1}^{n}\right)^{\text {geo }, p}$ (resp. $\widehat{\pi}_{1}\left(\mathcal{F}_{K} \backslash\right.$ $\left.S)^{\text {geo, } p}\right)$ for the maximal pro- $p$ quotient of $\widehat{\pi}_{1}\left(X_{K} \backslash\left(S \cup\left\{\infty_{i}\right\}_{i=1}^{n}\right) ;\left\{\infty_{i}\right\}_{i=1}^{n}\right)^{\text {geo }}$ (resp. $\left.\widehat{\pi}_{1}\left(\mathcal{F}_{K} \backslash S\right)^{\text {geo }}\right)$. Our first main result is the following (cf. Theorem 3.2).

Theorem 1. The (scheme) morphism $\mathcal{F}_{K} \rightarrow X_{K}$ induces a continuous homomorphism $\widehat{\pi}_{1}\left(\mathcal{F}_{K} \backslash S\right)^{\text {geo }} \rightarrow \widehat{\pi}_{1}\left(X_{K} \backslash\left(S \cup\left\{\infty_{i}\right\}_{i=1}^{n}\right) ;\left\{\infty_{i}\right\}_{i=1}^{n}\right)^{\text {geo }}$ (resp. $\widehat{\pi}_{1}\left(\mathcal{F}_{K} \backslash\right.$ $\left.S)^{\text {geo }, p} \rightarrow \widehat{\pi}_{1}\left(X_{K} \backslash\left(S \cup\left\{\infty_{i}\right\}_{i=1}^{n}\right) ;\left\{\infty_{i}\right\}_{i=1}^{n}\right)^{\text {geo }, p}\right)$ which makes $\widehat{\pi}_{1}\left(\mathcal{F}_{K} \backslash S\right)^{\text {geo }}$ (resp. $\left.\widehat{\pi}_{1}\left(\mathcal{F}_{K} \backslash S\right)^{\text {geo,p }}\right)$ into a semi-direct factor (cf. Definition 1.1.4 and Lemma 1.1.5) of $\widehat{\pi}_{1}\left(X_{K} \backslash\left(S \cup\left\{\infty_{i}\right\}_{i=1}^{n}\right) ;\left\{\infty_{i}\right\}_{i=1}^{n}\right)^{\text {geo }}\left(\right.$ resp. $\left.\widehat{\pi}_{1}\left(X_{K} \backslash\left(S \cup\left\{\infty_{i}\right\}_{i=1}^{n}\right) ;\left\{\infty_{i}\right\}_{i=1}^{n}\right)^{\text {geo }, p}\right)$. In particular, the above homomorphisms are injective.

In the course of proving Theorem 1 (cf. proof of Theorem 3.2) we prove the following.

Theorem 2. Let $f: \mathcal{Y} \rightarrow \mathcal{F}$ be a finite (Galois) covering with $\mathcal{Y}$ normal and geometrically connected, which is étale above $\mathcal{F}_{K} \backslash S$ and above the generic points of $\mathcal{F}_{k}$. Then there exists, after possibly a finite extension of $K$, a finite (Galois) covering $\tilde{f}: Y \rightarrow X$ of formal schemes with $Y$ normal and geometrically connected, which is étale above $X_{K} \backslash\left(S \cup\left\{\infty_{i}\right\}_{i=1}^{n}\right)$ and above the generic points of $X_{k}$, is possibly ramified above the points $\left\{\infty_{i}\right\}_{i=1}^{n}$ with ramification indices prime-to-p, and which induces by pull back via the (scheme) morphism $\mathcal{F} \rightarrow X$ the covering $f$.

Let $g_{x} \stackrel{\text { def }}{=} \operatorname{genus}\left(X_{K}\right)$, which is also called the genus of the formal fibre $\mathcal{F}_{K}$. Write $\pi_{1}\left(\mathcal{F}_{K} \backslash S, \eta\right)^{\text {geo }, p^{\prime}}\left(\right.$ resp. $\left.\pi_{1}\left(X_{K} \backslash\left(S \cup\left\{\infty_{i}\right\}_{i=1}^{n}\right), \eta\right)^{\text {geo }, p^{\prime}}\right)$ for the maximal prime-to- $p$ quotient of the geometric étale fundamental group $\pi_{1}\left(\mathcal{F}_{K} \backslash S, \eta\right)^{\text {geo }}$ (resp. $\left.\pi_{1}\left(X_{K} \backslash\left(S \cup\left\{\infty_{i}\right\}_{i=1}^{n}\right), \eta\right)^{\text {geo }}\right)$. Our second main result is the following (cf. Theorem $3.4)$. 
Theorem 3. Let $S(\bar{K})=\left\{y_{1}, \ldots, y_{m}\right\}$ of cardinality $m \geq 0$. Then the continuous homomorphism $\pi_{1}\left(\mathcal{F}_{K} \backslash S, \eta\right)^{\text {geo, } p^{\prime}} \rightarrow \pi_{1}\left(X_{K} \backslash\left(S \cup\left\{\infty_{i}\right\}_{i=1}^{n}\right), \eta\right)^{\text {geo, } p^{\prime}}$ (induced by the (scheme) morphism $\left.\mathcal{F}_{K} \rightarrow X_{K}\right)$ is an isomorphism. In particular, $\pi_{1}\left(\mathcal{F}_{K} \backslash\right.$ $S, \eta)^{\text {geo, } p^{\prime}}$ is (pro-)prime-to-p free of rank $2 g_{x}+n+m-1$ and can be generated by $2 g_{x}+n+m$ generators $\left\{a_{1}, \ldots, a_{g}, b_{1}, \cdots, b_{g}, \sigma_{1}, \ldots, \sigma_{n}, \tau_{1}, \ldots, \tau_{m}\right\}$ subject to the unique relation $\prod_{i=1}^{g}\left[a_{i}, b_{i}\right] \prod_{j=1}^{n} \sigma_{j} \prod_{t=1}^{m} \tau_{t}=1$, where $\sigma_{j}$ (resp $\tau_{t}$ ) is a generator of inertia at $\infty_{i}$ (resp. $\left.y_{t}\right)$.

Next, we outline the content of the paper. In $\S 1$ we collect some well-known background material. In $\S 2$ we investigate a certain quotient of the absolute Galois group of a formal boundary of a formal germ of a $p$-adic curve and prove Proposition 2.5 which is used in the proof of Theorem 1. In $\S 3$ we prove Theorems 1 and 3.

Notations. In this paper $K$ is a complete discrete valuation field, $R$ its valuation ring, $\pi$ a uniformising parameter, and $k \stackrel{\text { def }}{=} R / \pi R$ the residue field which we assume to be algebraically closed of characteristic $p \geq 0$.

We refer to [Raynaud], 3, for the terminology we will use concerning $K$-rigid analytic spaces, $R$-formal schemes, as well as the link between formal and rigid geometry. For an $R$-(formal) scheme $X$ we will denote by $X_{K} \stackrel{\text { def }}{=} X \times_{R} K$ (resp. $X_{k} \stackrel{\text { def }}{=} X \times_{R} k$ ) the generic (resp. special) fibre of $X$ (the generic fibre is understood in the rigid analytic sense in the case where $X$ is a formal scheme). Moreover, if $X=$ Spf $A$ is an affine formal $R$-scheme of finite type we denote by $X_{K} \stackrel{\text { def }}{=} \operatorname{Sp}\left(A \otimes_{R} K\right)$ the associated $K$-rigid affinoid space and will also denote, when there is no risk of confusion, by $X_{K}$ the affine scheme $X_{K} \stackrel{\text { def }}{=} \operatorname{Spec}\left(A \otimes_{R} K\right)$.

A formal (resp. algebraic) $R$-curve is an $R$-formal scheme of finite type (resp. $R$-scheme of finite type) flat, separated, and whose special fibre is equi-dimensional of dimension 1. For a $K$-scheme (resp. $K$-rigid analytic space) $X$ and $L / K$ a field extension (resp. a finite extension) we write $X_{L} \stackrel{\text { def }}{=} X \times_{K} L$ which is an $L$-scheme (resp. an $L$-rigid analytic space). If $X$ is a proper and normal formal $R$-curve we also denote, when there is no risk of confusion, by $X$ the algebraisation of $X$ which is an algebraic $R$-curve and by $X_{K}$ the proper normal and algebraic $K$-curve associated to the rigid $K$-curve $X_{K}$ via the rigid GAGA functor.

For a profinite group $H$ and a prime integer $\ell$ we denote by $H^{\ell}$ the maximal pro- $\ell$ quotient of $H$, and $H^{\ell^{\prime}}$ the maximal prime-to- $\ell$ quotient of $H$.

$\S 1$ Background. In this section we collect some background material used in this paper.

1.1. Let $p>1$ be a prime integer. We recall some well-known facts on profinite pro- $p$ groups. First, we recall the following characterisations of free pro- $p$ groups.

Proposition 1.1.1. Let $G$ be a profinite pro-p group. Then the following properties are equivalent.

(i) $G$ is a free pro-p group.

(ii) The p-cohomological dimension of $G$ satisfies $\operatorname{cd}_{p}(G) \leq 1$.

In particular, a closed subgroup of a free pro-p group is free.

Proof. Well-known (cf. [Serre], and [Ribes-Zalesskii], Theorem 7.7.4).

Next, we recall the notion of a direct factor of a free pro- $p$ group (cf. [Garuti], 1, the discussion preceding Proposition 1.8, and [Saïdi], §1). 
Definition/Lemma 1.1.2 (Direct factors of free pro- $p$ groups). Let $F$ be a free pro-p group, $H \subseteq F$ a closed subgroup, and $\iota: H \rightarrow F$ the natural homomorphism. We say that $H$ is a direct factor of $F$ if there exists a continuous homomorphism $s: F \rightarrow H$ such that $s \circ \iota=\mathrm{id}_{H}$. There exists then a (non unique) closed subgroup $N$ of $F$ such that $F$ is isomorphic to the free direct product $H \star N$. We will refer to such a subgroup $N$ as a supplement of $H$.

Proof. See [Saïdi] Lemma 1.1.2.

One has the following cohomological characterisation of direct factors of free pro- $p$ groups.

Proposition 1.1.3. Let $H$ be a pro-p group, $F$ a free pro-p group, and $\sigma: H \rightarrow F$ a continuous homomorphism. Assume that the map induced by $\sigma$ on cohomology

$$
h^{1}(\sigma): H^{1}(F, \mathbb{Z} / p \mathbb{Z}) \rightarrow H^{1}(H, \mathbb{Z} / p \mathbb{Z})
$$

is surjective, where $\mathbb{Z} / p \mathbb{Z}$ is considered as a trivial discrete module. Then $\sigma$ induces an isomorphism $H \stackrel{\sim}{\rightarrow} \sigma(H)$ and $\sigma(H)$ is a direct factor of $F$. In particular, $H$ is pro-p free. We say that $\sigma$ makes $H$ into a direct factor of $F$.

Proof. cf. [Garuti], Proposition 1.8 and Proposition 1.1.1 above.

Next, we consider the notion of a semi-direct factor of a profinite group.

Definition 1.1.4 (Semi-direct factors of profinite groups). Let $G$ be a profinite group, $H \subseteq G$ a closed subgroup, and $\iota: H \rightarrow G$ the natural homomorphism. We say that $H$ is a semi-direct factor of $G$ if there exists a continuous homomorphism $s: G \rightarrow H$ such that $s \circ \iota=\operatorname{id}_{H}$ ( $s$ is necessarily surjective).

Lemma 1.1.5. Let $\tau: H \rightarrow G$ be a continuous homomorphism between profinite groups. Write $H=\varliminf_{j \in J}^{\lim _{j}} H_{j}$ as the projective limit of the inverse system $\left\{H_{j}, \phi_{j^{\prime} j}, J\right\}$ of finite quotients $H_{j}$ of $H$ with index set $J$. Suppose there exists, $\forall j \in J, a$ surjective homomorphism $\psi_{j}: G \rightarrow H_{j}$ such that $\tau \circ \psi_{j}: H \rightarrow H_{j}$ is the natural map and $\psi_{j}=\phi_{j^{\prime} j} \circ \psi_{j^{\prime}}$ whenever this makes sense. Then $\tau$ induces an isomorphism $H \stackrel{\sim}{\rightarrow} \tau(H)$ and $\tau(H)$ is a semi-direct factor of $G$. We say that $\tau$ makes $H$ into a semi-direct factor of $G$.

Proof. Indeed, the $\left\{\psi_{j}\right\}_{j \in J}$ give rise to a continuous (necessarily surjective) homomorphism $\psi: G \rightarrow H$ which is a right inverse of $\tau$.

1.2. Formal Patching. Next, we explain the procedure which allows to construct (Galois) coverings of curves in the setting of formal geometry by patching coverings of formal (affine, non-proper) curves with coverings of formal germs at closed points of the special fibre along the boundaries of these formal germs.

1.2.1. Let $X$ be a proper, normal, formal $R$-curve with $X_{k}$ reduced. For $x \in X$ a closed point let $\mathcal{F}_{x} \stackrel{\text { def }}{=} \operatorname{Spf} \hat{\mathcal{O}}_{X, x}$ be the formal completion of $X$ at $x$ which we will refer to as the formal germ of $X$ at $x$. Thus, $\hat{\mathcal{O}}_{X, x}$ is the completion of the local ring of the algebraisation of $X$ at $x$. Write $\mathcal{F}_{x, K} \stackrel{\text { def }}{=} \operatorname{Spec}\left(\hat{\mathcal{O}}_{X, x} \otimes_{R} K\right)$. We will refer to $\mathcal{F}_{x, K}$ as the formal fibre of $X_{K}$ at $x$. Let $\left\{\mathcal{P}_{i}\right\}_{i=1}^{n}$ be the minimal prime ideals of $\hat{\mathcal{O}}_{X, x}$ which contain $\pi$; they correspond to the branches $\left\{\eta_{i}\right\}_{i=1}^{n}$ of the completion of $X_{k}$ at $x$ (i.e., closed points of the normalisation of $X_{k}$ above $x$ ), and 
$\mathcal{X}_{i}=\mathcal{X}_{x, i} \stackrel{\text { def }}{=} \operatorname{Spf} \hat{\mathcal{O}}_{x, \mathcal{P}_{i}}$ the formal completion of the localisation of $\mathcal{F}_{x}$ at $\mathcal{P}_{i}$. The local ring $\hat{\mathcal{O}}_{x, \mathcal{P}_{i}}$ is a complete discrete valuation ring with uniformiser $\pi$. We refer to $\left\{\mathcal{X}_{i}\right\}_{i=1}^{n}$ as the set of boundaries of the formal germ $\mathcal{F}_{x}$. We have a canonical morphism $\mathcal{X}_{i} \rightarrow \mathcal{F}_{x}$ of formal schemes, $1 \leq i \leq n$.

Let $Z$ be a finite set of closed points of $X$ and $U \subset X$ a formal sub-scheme of $X$ whose special fibre is $U_{k} \stackrel{\text { def }}{=} X_{k} \backslash Z$.

Definition 1.2.2. We use the notations above. A $(G$-)covering patching data for the pair $(X, Z)$ consists of the following.

(i) A finite (Galois) covering $V \rightarrow U$ of formal schemes (with Galois group $G$ ).

(ii) For each point $x \in Z$, a finite (Galois) covering $\mathcal{Y}_{x} \rightarrow \mathcal{F}_{x}$ of formal schemes (with Galois group $G$ ).

The above data (i) and (ii) must satisfy the following compatibility condition. (iii) If $\left\{\mathcal{X}_{i}\right\}_{i=1}^{n}$ are the boundaries of the formal germ at the point $x$, then for $1 \leq i \leq n$ is given a ( $G$-equivariant) $\mathcal{X}_{i}$-isomorphism

$$
\mathcal{Y}_{x} \times \mathcal{F}_{x} \mathcal{X}_{i} \stackrel{\sim}{\rightarrow} V \times_{U} \mathcal{X}_{i}
$$

Property (iii) should hold for each $x \in Z$. (Note that there are natural morphisms $\mathcal{X}_{i} \rightarrow U$ of formal schemes, $1 \leq i \leq n$.)

The following is the main patching result that we will use in this paper (cf. [Pries], Theorem 3.4, [Harbater], Theorem 3.2.8).

Proposition 1.2.3. We use the notations above. Given a $(G$-) covering patching data as in Definition 1.2.2 there exists a unique, up to isomorphism, (Galois) covering $Y \rightarrow X$ of formal schemes (with Galois group $G$ ) which induces the above $(G$-)covering in Definition 1.2.2(i) when restricted to $U$, and induces the above $(G-)$ covering in Definition 1.2.2(ii) when pulled-back to $\mathcal{F}_{x}$ for each point $x \in Z$.

1.2.4. With the same notations as above, let $x \in X$ be a closed point and $\widetilde{X}_{k}$ the normalisation of $X_{k}$. There is a one-to-one correspondence between the set of points of $\widetilde{X}_{k}$ above $x$ and the set of boundaries of the formal germ of $X$ at the point $x$. Let $x_{i}$ be the point of $\widetilde{X}_{k}$ above $x$ which corresponds to the boundary $\mathcal{X}_{i}$, $1 \leq i \leq n$. Then the completion of $\widetilde{X}_{k}$ at $x_{i}$ is isomorphic to the spectrum of a ring of formal power series $k\left[\left[t_{i}\right]\right]$ over $k$ where $t_{i}$ is a local parameter at $x_{i}$. The complete local ring $\hat{\mathcal{O}}_{x, \mathcal{P}_{i}}$ is a discrete valuation ring with uniformiser $\pi$ and residue field isomorphic to $k\left(\left(t_{i}\right)\right)$. Fix an isomorphism $k\left(\left(t_{i}\right)\right) \stackrel{\sim}{\rightarrow} \hat{\mathcal{O}}_{x, \mathcal{P}_{i}} / \pi$. Let $T_{i} \in \hat{\mathcal{O}}_{x, \mathcal{P}_{i}}$ be an element which lifts (the image in $\hat{\mathcal{O}}_{x, \mathcal{P}_{i}} / \pi$ under the above isomorphism of) $t_{i}$; we shall refer to such an element $T_{i}$ as a parameter of $\hat{\mathcal{O}}_{x, \mathcal{P}_{i}}$, or of the boundary $\mathcal{X}_{i}$. Then there exists an isomorphism $R\left[\left[T_{i}\right]\right]\left\{T_{i}^{-1}\right\} \stackrel{\sim}{\rightarrow} \hat{\mathcal{O}}_{x, \mathcal{P}_{i}}$, where

$$
R[[T]]\left\{T^{-1}\right\} \stackrel{\text { def }}{=}\left\{\sum_{i=-\infty}^{\infty} a_{i} T^{i}, \lim _{i \rightarrow-\infty}\left|a_{i}\right|=0\right\}
$$

and | | is a normalised absolute value of $R$ (cf. [Bourbaki], §2, 5).

1.3. Let $X$ be a normal and geometrically connected flat $R$-scheme (resp. $R$-formal affine scheme) whose special fibre is equidimensional of dimension $1, F \subset X_{K}$ a finite set of closed points, and $\eta$ a geometric point of $X$ above its generic point. 
Then $\eta$ determines an algebraic closure $\bar{K}$ of $K$ and we have an exact sequence of arithmetic fundamental groups

$$
1 \rightarrow \pi_{1}\left(X_{K} \backslash F, \eta\right)^{\mathrm{geo}} \rightarrow \pi_{1}\left(X_{K} \backslash F, \eta\right) \rightarrow \operatorname{Gal}(\bar{K} / K) \rightarrow 1
$$

where $\pi_{1}\left(X_{K} \backslash F, \eta\right)^{\text {geo }} \stackrel{\text { def }}{=} \operatorname{Ker}\left(\pi_{1}\left(X_{K} \backslash F, \eta\right) \rightarrow \operatorname{Gal}(\bar{K} / K)\right)$ is the geometric fundamental group of $X_{K}$ with generic point $\eta$. (In case $X=\operatorname{Spf} A$ is formal affine we define $\pi_{1}\left(X_{K}, \eta\right) \stackrel{\text { def }}{=} \pi_{1}\left(\operatorname{Spec} A_{K}, \eta\right)$ and similarly we define $\pi_{1}\left(X_{K} \backslash F, \eta\right)$, cf. [Saïdi], 2.1.)

Definition 1.3.1. Let $S, T \subset X_{K}$ be (possibly empty) finite sets of closed points (which we also view as reduced closed sub-schemes of $X_{K}$ ). Assume that the special fibre $X_{k}$ of $X$ is reduced. Let $I \stackrel{\text { def }}{=} I_{X_{k}, T} \subset \pi_{1}\left(X_{K} \backslash(S \cup T), \eta\right)^{\text {geo }}$ be the subgroup normally generated by the inertia subgroups above the generic points of $X_{k}$ and the pro- $p$ Sylow subgroups of the inertia groups above all points in $T$. We define

$$
\widehat{\pi}_{1}\left(X_{K} \backslash(S \cup T) ; T, \eta\right)^{\text {geo }} \stackrel{\text { def }}{=} \pi_{1}\left(X_{K} \backslash(S \cup T), \eta\right)^{\text {geo }} / I
$$

and refer to it as the geometric étale fundamental group of $X_{K} \backslash(S \cup T)$; with base point $\eta$, generically étale above $X_{k}$ and tamely ramified above $T$. In case $T=\emptyset$ and $U_{K} \stackrel{\text { def }}{=} X_{K} \backslash S$ we simply write $\widehat{\pi}_{1}\left(U_{K}, \eta\right)^{\text {geo }} \stackrel{\text { def }}{=} \widehat{\pi}_{1}\left(X_{K} \backslash S ; \emptyset, \eta\right)^{\text {geo }}$.

Note that the definition of $\widehat{\pi}_{1}\left(X_{K} \backslash(S \cup T) ; T, \eta\right)^{\text {geo }}$ depends on the model $X$ of $X_{K}$ (the model $X$ of $X_{K}$ will be fixed in later discussions in this paper). The profinite group $\widehat{\pi}_{1}\left(X_{K} \backslash(S \cup T) ; T, \eta\right)^{\text {geo }}$ classifies finite covers $f: Y_{L} \rightarrow X_{L} \stackrel{\text { def }}{=}$ $X \times_{K} L$ where $L / K$ is a finite extension with valuation ring $R_{L}$, which are étale above $X_{L} \backslash(S \cup T)_{L}$ (here $\left.(S \cup T)_{L} \stackrel{\text { def }}{=}(S \cup T) \times_{K} L\right)$ and possibly ramified with ramification indices prime-to- $p$ above the points in $T_{L} \stackrel{\text { def }}{=} T \times_{K} L, Y_{L}$ is geometrically connected, and such that $f$ extends after possibly a finite extension of $L$ to a finite cover $\tilde{f}: Y \rightarrow X_{R_{L}} \stackrel{\text { def }}{=} X \times_{R} R_{L}$ with $Y$ normal and $\tilde{f}$ is étale above the generic points of $X_{k}$. Note that if $X$ is a smooth $R$-formal affine scheme as above which is an $R$-formal curve then $\widehat{\pi}_{1}\left(X_{K}, \eta\right)^{\text {geo }}$ is isomorphic to the geometric étale fundamental group of the affine scheme $X_{k}$ as follows from the theorems of liftings of étale coverings (cf. [Grothendieck], Exposé I, Corollaire 8.4) and the theorem of purity of Zarizski-Nagata (cf. loc. cit. Exposé X, Théorème de pureté 3.1). Note also that $\widehat{\pi}_{1}\left(X_{K} \backslash(S \cup T) ; T, \eta\right)^{\text {geo, } p^{\prime}}=\pi_{1}\left(X_{K} \backslash(S \cup T), \eta\right)^{\text {geo, } p^{\prime}}$, as follows easily from Abhyankar's lemma (cf. loc. cit. Exposé X, Lemme 3.6).

$\S 2$. Geometric Galois groups of formal boundaries of formal germs of $p$-adic formal curves. In this section we investigate the structure of a certain quotient of the geometric Galois group of a formal boundary of a formal germ of a formal $R$-curve. The results in this section will be used in $\S 3$.

Let $D \stackrel{\text { def }}{=}$ Spf $R\left\langle\frac{1}{T}\right\rangle$ be the formal standard $R$-closed unit disc and $D_{K} \stackrel{\text { def }}{=}$ Sp $K\left\langle\frac{1}{T}\right\rangle$ its generic fibre which is the standard rigid $K$-closed unit disc centred at $\infty$. Write $\mathcal{X}=\operatorname{Spf} R[[T]]\left\{T^{-1}\right\}$ and $\mathcal{X}_{K} \stackrel{\text { def }}{=} \operatorname{Spec}\left(R[[T]]\left\{T^{-1}\right\} \otimes_{R} K\right)$. We have natural morphisms $\mathcal{X} \rightarrow D$ of formal $R$-schemes, and $\mathcal{X}_{K} \rightarrow D_{K}$ of $K$-schemes (cf. Notations). Let $\eta$ be a geometric point of $\mathcal{X}_{K}$ with value in its generic point which 
determines a generic point of $D_{K}$; which we denote also $\eta$, as well as algebraic closures $\bar{K}$ of $K, \bar{k}$ of $k$, and $\bar{M}$ of $M \stackrel{\text { def }}{=} \operatorname{Fr}\left(R[[T]]\left\{T^{-1}\right\}\right)$. We have an exact sequence of Galois groups

$$
1 \rightarrow \operatorname{Gal}(\bar{M} / \bar{K} \cdot M) \rightarrow \operatorname{Gal}(\bar{M} / M) \rightarrow \operatorname{Gal}(\bar{K} / K) \rightarrow 1 .
$$

Let $I \stackrel{\text { def }}{=} I_{\left(\mathcal{X}_{k}\right)} \subset \operatorname{Gal}(\bar{M} / \bar{K} \cdot M)$ be the subgroup normally generated by the inertia subgroups above the generic point of $\mathcal{X}_{k}$. Write $\Delta \stackrel{\text { def }}{=} \operatorname{Gal}(\bar{M} / \bar{K} \cdot M) / I$ and $\Gamma \stackrel{\text { def }}{=} \Delta^{p^{\prime}}$. We have an exact sequence

$$
1 \rightarrow P \rightarrow \Delta \rightarrow \Gamma \rightarrow 1
$$

where $P \stackrel{\text { def }}{=} \operatorname{Ker}(\Delta \rightarrow \Gamma)$.

Lemma 2.1. With the notations above, $P$ is the unique pro-p Sylow subgroup of $\Delta$, $P$ is pro-p free, and $\Gamma$ is canonically isomorphic to the maximal prime-to-p quotient $\hat{\mathbb{Z}}(1)^{p^{\prime}}$ of the Tate twist $\hat{\mathbb{Z}}(1)$.

Proof. Indeed, it follows from the various Definitions that $\Delta$ is isomorphic to the absolute Galois group of $\bar{k}((t))$ which is known to be an extension of $\hat{\mathbb{Z}}(1)^{p^{\prime}}$ by a free pro- $p$ group.

Lemma 2.2. Assume $p>0$. Then the pro-p group $\widehat{\pi}_{1}\left(D_{K}, \eta\right)^{\text {geo, } p}$ is free.

Proof. Indeed, it follows from the various Definitions that $\widehat{\pi}_{1}\left(D_{K}, \eta\right)^{\text {geo, } p}$ is isomorphic to the maximal pro- $p$ quotient of the geometric fundamental group of $D_{k}=\mathbb{A}_{k}^{1}$ which is pro- $p$ free (cf. [Serre1], Proposition 1).

Proposition 2.3. Assume $p>0$. Then the homomorphism $\Delta \rightarrow \widehat{\pi}_{1}\left(D_{K}, \eta\right)^{\text {geo }}$ induced by the morphism $\mathcal{X}_{K} \rightarrow D_{K}$ induces a homomorphism $\Delta^{p} \rightarrow \widehat{\pi}_{1}\left(D_{K}, \eta\right)^{\text {geo }, p}$ which makes $\Delta^{p}$ into a direct factor of $\widehat{\pi}_{1}\left(D_{K}, \eta\right)^{\text {geo, } p}$. Moreover, $\Delta^{p}$ is a free pro- $p$ group of infinite rank.

Proof. We show that the map $\psi: H^{1}\left(\widehat{\pi}_{1}\left(D_{K}, \eta\right)^{\text {geo }}, \mathbb{Z} / p \mathbb{Z}\right) \rightarrow H^{1}(\Delta, \mathbb{Z} / p \mathbb{Z})$ induced by the homomorphism $\Delta \rightarrow \widehat{\pi}_{1}\left(D_{K}, \eta\right)^{\text {geo }}$ on cohomology is surjective (cf. Proposition 1.1.3). Let $\tilde{f}: \Delta \rightarrow \mathbb{Z} / p \mathbb{Z}$ be a surjective homomorphism and $f: \mathcal{Y} \rightarrow \mathcal{X}$ the corresponding Galois cover (which we can assume, without loss of generality, defined over $K$ ) with $\mathcal{Y}$ normal, geometrically connected, and $f$ is étale above the generic point of $\mathcal{X}_{k}$ (hence $f$ is étale above $\mathcal{X}$ ). Thus, $f_{k}: \mathcal{Y}_{k} \rightarrow \mathcal{X}_{k}=\operatorname{Spec} k((t))$ is an étale $\mathbb{Z} / p \mathbb{Z}$-torsor. By Artin-Schreier theory the torsor $f_{k}$ can be approximated by a Galois cover $g_{k}: Y_{k} \rightarrow \mathbb{P}_{k}^{1}$ of degree $p$ which is étale outside the point $t=0$ and whose completion above this point is isomorphic to $f_{k}$. The étale $\mathbb{Z} / p \mathbb{Z}$-torsor $g_{k}^{-1}\left(\mathbb{A}_{k}^{1}\right) \rightarrow \mathbb{A}_{k}^{1} \stackrel{\text { def }}{=} \mathbb{P}_{k}^{1} \backslash\{t=0\}=$ Spec $k\left[\frac{1}{t}\right]$ lifts (uniquely up to isomorphism) to an étale $\mathbb{Z} / p \mathbb{Z}$-torsor $g: Z_{K} \rightarrow D_{K}$ by the theorems of liftings of étale covers (cf. [Grothendieck], Exposé I, Corollaire 8.4) which gives rise to a class in $H^{1}\left(\widehat{\pi}_{1}\left(D_{K}, \eta\right)^{\text {geo }}, \mathbb{Z} / p \mathbb{Z}\right)$ that is easily verified to map to the class of $f$ in $H^{1}(\Delta, \mathbb{Z} / p \mathbb{Z})$. Moreover, $\Delta^{p}$ has infinite rank as it is isomorphic to the maximal pro- $p$ quotient of the absolute Galois group of $\bar{k}((t))$ which is known to be free of infinite rank.

Write $\widetilde{\Gamma} \stackrel{\text { def }}{=} \widehat{\pi}_{1}\left(D_{K} \backslash\{\infty\} ;\{\infty\}, \eta\right)^{\text {geo, } p^{\prime}}=\pi_{1}\left(D_{K} \backslash\{\infty\}, \eta\right)^{\text {geo, } p^{\prime}}$ (cf. 1.3) for the maximal prime-to- $p$ quotient of $\widehat{\pi}_{1}\left(D_{K} \backslash\{\infty\} ;\{\infty\}, \eta\right)^{\text {geo }}$. 
Lemma 2.4. The morphism $\mathcal{X}_{K} \rightarrow D_{K}$ induces a canonical homomorphism $\Gamma \rightarrow \widetilde{\Gamma}$ which is an isomorphism. In particular, $\widetilde{\Gamma}$ is (canonically) isomorphic to $\hat{\mathbb{Z}}(1)^{p^{\prime}}$.

Proof. Follows easily from the fact that a Galois covering $Y_{K} \rightarrow D_{K}$ of order primeto- $p$ with $Y_{K}$ geometrically connected, ramified only above $\infty$ is, possibly after a finite extension of $K$ and for a suitable choice of the parameter $T$ of $D_{K}$, generically a $\mu_{n}$-torsor given generically by the equation $S^{n}=T$ for some positive integer $n$ prime-to- $p$.

Consider the following exact sequence

$$
1 \rightarrow \mathcal{H} \rightarrow \widehat{\pi}_{1}\left(D_{K} \backslash\{\infty\} ;\{\infty\}, \eta\right)^{\text {geo }} \rightarrow \widetilde{\Gamma} \rightarrow 1,
$$

where $\mathcal{H} \stackrel{\text { def }}{=} \operatorname{Ker}\left(\widehat{\pi}_{1}\left(D_{K} \backslash\{\infty\} ;\{\infty\}, \eta\right)^{\text {geo }} \rightarrow \widetilde{\Gamma}\right)$. Further, let $\widetilde{P} \stackrel{\text { def }}{=} \mathcal{H}^{p}$ be the maximal pro- $p$ quotient of $\mathcal{H}$. By pushing out the above sequence by the (characteristic) quotient $\mathcal{H} \rightarrow \widetilde{P}$ we obtain an exact sequence

$$
1 \rightarrow \widetilde{P} \rightarrow \widetilde{\Delta} \rightarrow \widetilde{\Gamma} \rightarrow 1
$$

Proposition 2.5. The morphism $\mathcal{X}_{K} \rightarrow D_{K}$ induces a commutative diagram of exact sequences

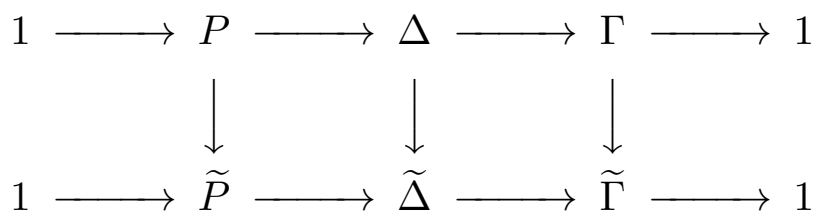

where the right vertical homomorphism $\Gamma \rightarrow \widetilde{\Gamma}$ is an isomorphism (cf. Lemma 2.4) and the middle vertical homomorphism $\Delta \rightarrow \widetilde{\Delta}$ makes $\Delta$ into a semi-direct factor of $\widetilde{\Delta}$ (cf. Lemma 1.1.5).

Proof. Let $\Delta \rightarrow G$ be a finite quotient which sits in an exact sequence $1 \rightarrow Q \rightarrow$ $G \rightarrow \Gamma_{n} \rightarrow 1$ where $\Gamma_{n}$ is the unique quotient of $\Gamma$ of cardinality $n$; for some integer $n$ prime-to- $p$, with $Q$ a $p$-group (cf. Lemma 2.1). We will show there exists a surjective homomorphism $\widetilde{\Delta} \rightarrow G$ whose composition with $\Delta \rightarrow \widetilde{\Delta}$ is the above homomorphism. We can assume, without loss of generality, that the corresponding Galois covering $f: \mathcal{Y} \rightarrow \mathcal{X}$ with group $G$ is defined over $K, \mathcal{Y}$ is normal and connected, and $f$ is étale. This covering factorises as $\mathcal{Y} \rightarrow \mathcal{X}^{\prime} \rightarrow \mathcal{X}$ where $\mathcal{X}^{\prime} \rightarrow \mathcal{X}$ is Galois with group $\Gamma_{n} \stackrel{\sim}{\rightarrow} \mu_{n}$ and $\mathcal{Y} \rightarrow \mathcal{X}^{\prime}$ is Galois with group $Q$. After possibly a finite extension of $K$ the $\mu_{n}$-torsor $\mathcal{X}^{\prime} \rightarrow \mathcal{X}$ extends to a generically $\mu_{n}$-torsor $D^{\prime} \rightarrow D$ defined generically by an equation $S^{n}=T$, for a suitable choice of the parameter $T$ of $D$, which is (totally) ramified only above $\infty, D^{\prime}=\operatorname{Spf} R\left\langle\frac{1}{S}\right\rangle$ is a closed formal unit disc centred at the unique point; which we denote also $\infty$, above $\infty \in D$ and $\mathcal{X}^{\prime}=\operatorname{Spf} R[[S]]\left\{S^{-1}\right\}$ (cf. Lemma 2.4 and the isomorphism $\Gamma \stackrel{\sim}{\rightarrow} \widetilde{\Gamma}$ therein).

For the rest of the proof we assume $p>0$. By Proposition 2.3, applied to $\mathcal{X}^{\prime} \rightarrow D^{\prime}$, there exists (after possibly a finite extension of $K$ ) an étale Galois covering $Y \rightarrow D^{\prime}$ with group $Q, Y$ is normal and geometrically connected, and such that we have a commutative diagram of cartesian squares

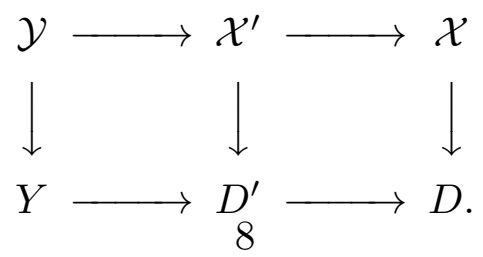


Next, we borrow some ideas from [Garuti] (preuve du Théorème 2.13). We claim one can choose the above (geometric) covering $Y \rightarrow D^{\prime}$ such that the finite composite covering $Y \rightarrow D$ is Galois with group $G$. Indeed, consider the quotient $\Delta \rightarrow \Delta_{\mathcal{X}^{\prime}}$ (resp. $\widetilde{\Delta} \rightarrow \widetilde{\Delta}_{D^{\prime}}$ ) of $\Delta$ (resp. $\widetilde{\Delta}$ ) which sits in the following exact sequence $1 \rightarrow P_{\mathcal{X}^{\prime}} \rightarrow \Delta_{\mathcal{X}^{\prime}} \rightarrow \Gamma_{n} \rightarrow 1$ where $P_{\mathcal{X}^{\prime}} \stackrel{\text { def }}{=} \widehat{\pi}_{1}\left(\mathcal{X}^{\prime}, \eta\right)^{\text {geo,p }}\left(\right.$ resp. $1 \rightarrow \widetilde{P}_{D^{\prime}} \rightarrow \widetilde{\Delta}_{D^{\prime}} \rightarrow$ $\widetilde{\Gamma}_{n} \rightarrow 1$ where $\left.\widetilde{P}_{D^{\prime}} \stackrel{\text { def }}{=} \widehat{\pi}_{1}\left(D^{\prime}, \eta\right)^{\text {geo,p }}\right)$. We have a commutative diagram of exact sequences

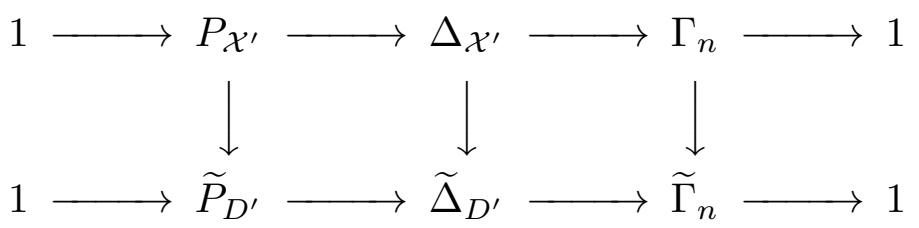

where the right vertical map is an isomorphism (cf. Lemma 2.4). The choice of a splitting of the upper sequence in the above diagram (which splits since $P_{\mathcal{X}^{\prime}}$ is pro- $p$ and $\Gamma_{n}$ is cyclic (pro-)prime-to- $p$ ) induces an action of $\Gamma_{n}$ on $\widetilde{P}_{D^{\prime}}$ and $P_{\mathcal{X}^{\prime}}$ is a direct factor of $\widetilde{P}_{D^{\prime}}$ (cf. Proposition 2.3) which is stable by this action of $\Gamma_{n}$. Further, $P_{\mathcal{X}^{\prime}}$ has a supplement $E$ in $\widetilde{P}_{D^{\prime}}$ which is invariant under the action of $\Gamma_{n}$ by [Garuti], Corollaire 1.11. The existence of this supplement $E$ implies that one can choose $Y \rightarrow D^{\prime}$ as above such that the finite composite covering $Y \rightarrow D$ is Galois with group $G$. More precisely, if the Galois covering $\mathcal{Y} \rightarrow \mathcal{X}^{\prime}$ corresponds to the surjective homomorphism $\rho: P_{\mathcal{X}^{\prime}} \rightarrow Q$ (which is stable by $\Gamma_{n}$ since $\mathcal{Y} \rightarrow \mathcal{X}$ is Galois) then we consider the Galois covering $Y \rightarrow D^{\prime}$ corresponding to the surjective homomorphism $\widetilde{P}_{D^{\prime}}=P_{\mathcal{X}^{\prime}} \star E \rightarrow Q$ which is induced by $\rho$ and the trivial homomorphism $E \rightarrow Q$, which is stable by $\Gamma_{n}$.

The above construction can be performed in a functorial way with respect to the various finite quotients of $\Delta$. More precisely, let $\left\{\phi_{j}: \Delta \rightarrow G_{j}\right\}_{j \in J}$ be a cofinal system of finite quotients of $\Delta$ where $G_{j}$ sits in an exact sequence $1 \rightarrow Q_{j} \rightarrow$ $G_{j} \rightarrow \Gamma_{n_{j}} \rightarrow 1$, for some integer $n_{j}$ prime-to- $p$, and $Q_{j}$ a $p$-group. Assume we have a factorisation $\Delta \rightarrow G_{j^{\prime}} \rightarrow G_{j}$ for $j^{\prime}, j \in J$. Thus, $n_{j}$ divides $n_{j^{\prime}}$, and we can assume without loss of generality (after replacing the group extension $G_{j}$ by its pull-back via $\Gamma_{n_{j}^{\prime}} \rightarrow \Gamma_{n_{j}}$ ) that $n \stackrel{\text { def }}{=} n_{j}=n_{j^{\prime}}$. With the above notations we then have surjective homomorphisms $\rho_{j^{\prime}}: P_{\mathcal{X}^{\prime}} \rightarrow Q_{j^{\prime}}, \rho_{j}: P_{\mathcal{X}^{\prime}} \rightarrow Q_{j}$ (which are stable by $\left.\Gamma_{n}\right)$, and $\rho_{j}$ factorises through $\rho_{j^{\prime}}$. Then we consider the Galois covering(s) $Y_{j^{\prime}} \rightarrow D^{\prime}$ (resp. $Y_{j} \rightarrow D^{\prime}$ ) corresponding to the surjective homomorphism(s) $\psi_{j^{\prime}}$ : $\widetilde{P}_{D^{\prime}}=P_{\mathcal{X}^{\prime}} \star E \rightarrow Q$ (resp. $\psi_{j}: \widetilde{P}_{D^{\prime}}=P_{\mathcal{X}^{\prime}} \star E \rightarrow Q$ ) which are induced by $\rho_{j^{\prime}}$ (resp. $\rho_{j}$ ) and the trivial homomorphism $E \rightarrow Q$, which are stable by $\Gamma_{n}$ and $\psi_{j}$ factorises through $\psi_{j^{\prime}}$. We deduce from this construction the existence of a surjective continuous homomorphism $\widetilde{\Delta} \rightarrow \Delta$ which is a right inverse to the natural homomorphism $\Delta \rightarrow \widetilde{\Delta}$ (cf. Lemma 1.1.5).

$\S 3$ Geometric fundamental groups of formal fibres of $p$-adic curves. In this section we investigate the structure of $\widehat{\pi}_{1}$ of a formal fibre of a $K$-curve. Let $X$ be a proper, normal, formal $R$-curve whose special fibre $X_{k}$ is reduced and consists of $n \geq 1$ distinct irreducible components $\left\{P_{i}\right\}_{i=1}^{n}$ which intersect at a (closed) point $x \in X_{k}(k)$, and $x$ is the unique singular point of $X_{k}$. Write $\widetilde{P}_{i} \rightarrow P_{i}$ for the morphism of normalisation. We assume $\widetilde{P}_{i}=\mathbb{P}_{k}^{1}$ is a projective line, the morphism $\widetilde{P}_{i} \rightarrow P_{i}$ is a homeomorphism, and if $x_{i}$ is the (unique) pre-image of $x$ 
in $\widetilde{P}_{i}$ then $x_{i} \in \widetilde{P}_{i}(k)$ is the zero point of $\widetilde{P}_{i}$. In particular, the configuration of the irreducible components of $X_{k}$ is tree-like. The formal curve $X$ has a formal covering $X=B \cup D_{1} \cup \ldots \cup D_{n}$ where $B \subset X$ is a formal sub-scheme with special fibre $B_{k}=X_{k} \backslash\left\{\infty_{i}\right\}_{i=1}^{n}, \infty_{i}$ is the image in $P_{i}$ of the infinity point of $\widetilde{P}_{i}$, $D_{i}=\operatorname{Spf} R\left\langle\frac{1}{T_{i}}\right\rangle$ is an $R$-formal closed unit disc with special fibre $D_{i, k}=P_{i} \backslash\{x\}$ and generic fibre $D_{i, K}=\operatorname{Sp} K\left\langle\frac{1}{T_{i}}\right\rangle$ which is a closed unit $K$-rigid disc centred at the point $\infty_{i} \in D_{i, K}(K)$ which specialises into the infinity point $\infty_{i} \in P_{i}$, $1 \leq i \leq n$. Write $\mathcal{F} \stackrel{\text { def }}{=} \mathcal{F}_{x}=\operatorname{Spf} \hat{\mathcal{O}}_{X, x}$ for the formal germ of $X$ at $x$ and $\mathcal{F}_{K} \stackrel{\text { def }}{=} \mathcal{F}_{x, K}=\operatorname{Spec}\left(\hat{\mathcal{O}}_{X, x} \otimes_{R} K\right)$ for the formal fibre of $X_{K}$ at $x$ (cf. 1.2.1). For $1 \leq i \leq n$, let $\mathcal{X}_{i}$ be the formal boundary of $\mathcal{F}$ corresponding to the point $x_{i}$ above. The completion of the normalisation $X_{k}^{\text {nor }}$ of $X_{k}$ at $x_{i}$ is isomorphic to the spectrum of a ring of formal power series $k\left[\left[t_{i}\right]\right]$ in one variable over $k$, and $\mathcal{X}_{i} \stackrel{\sim}{\rightarrow} \operatorname{Spf} R\left[\left[T_{i}\right]\right]\left\{T_{i}^{-1}\right\}$ (cf. 1.2.4).

Remark 3.1. Let $\widetilde{Y}$ be a proper and normal formal $R$-curve with $\widetilde{Y}_{k}$ reduced and $y \in \tilde{Y}(k)$ a closed point. Write $\mathcal{G} \stackrel{\text { def }}{=} \mathcal{G}_{y}=\operatorname{Spf} \hat{\mathcal{O}}_{\widetilde{Y}, y}$ for the formal germ of $\widetilde{Y}$ at $y$ and $\mathcal{G}_{K} \stackrel{\text { def }}{=} \mathcal{G}_{y, K}=\operatorname{Spec}\left(\hat{\mathcal{O}}_{\widetilde{Y}, y} \otimes_{R} K\right)$ for the formal fibre of $\widetilde{Y}_{K}$ at $y$ (cf. 1.2.1). Let $\left\{\mathcal{Y}_{i}\right\}_{i=1}^{n}$ be the set of formal boundaries of $\mathcal{G}$, and $y_{i} \in\left(\widetilde{Y}_{k}\right)^{\text {nor }}(k)$ the point of the normalisation $\left(\widetilde{Y}_{k}\right)^{\text {nor }}$ of $\widetilde{Y}_{k}$ above $y$ which corresponds to the boundary $\mathcal{Y}_{i}$, $1 \leq i \leq n$. The completion of $\left(\widetilde{Y}_{k}\right)^{\text {nor }}$ at $y_{i}$ is isomorphic to the spectrum of a ring of formal power series $k\left[\left[s_{i}\right]\right]$ in one variable over $k$ and $\mathcal{Y}_{i} \stackrel{\sim}{\rightarrow} \operatorname{Spf} R\left[\left[S_{i}\right]\right]\left\{S_{i}^{-1}\right\}$ (cf. 1.2.4). One can construct a compactification of $\mathcal{G}$ (as in the above discussion where $\mathcal{G}=\mathcal{F}$ ) which is a formal and proper $R$-curve $Y \stackrel{\text { def }}{=} Y_{y}$ obtained by patching an $R$-formal closed unit disc $Y_{i}=\operatorname{Spf} R\left\langle\frac{1}{S_{i}}\right\rangle$ with $\mathcal{G}$ along the boundary $\mathcal{Y}_{i}$, for $1 \leq i \leq n$. The resulting formal $R$-curve $Y$ has a special fibre $Y_{k}$ consisting of $n$ distinct reduced irreducible components $\left\{Q_{i}\right\}_{i=1}^{n}$ which intersect at the (closed) point $y$, and $y$ is the unique singular point of $Y_{k}$. Moreover, if we write $\widetilde{Q}_{i} \rightarrow Q_{i}$ for the morphism of normalisation then $\widetilde{Q}_{i}=\mathbb{P}_{k}^{1}$ is a projective line and the morphism $\widetilde{Q}_{i} \rightarrow Q_{i}$ is a homeomorphism. By construction the formal germ (resp. formal fibre) of $Y$ (resp. of $Y_{K}$ ) at the closed point $y$ is isomorphic to $\mathcal{G}$ (resp. $\mathcal{G}_{K}$ ). (cf. [Bosch-Lütkebohmert], Definition 4.4, for a rigid analytic construction of the generic fibre $Y_{K}^{\text {rig }} \stackrel{\text { def }}{=} Y_{K}$ of the above compactification $Y$ endowed with a formal covering corresponding to the above formal model $Y$ of $Y_{K}$, as well as [Bosch], Theorem 5.8, for the invariance of the formal germ at $y$ under this construction.)

Let $\eta$ be a geometric point of $\mathcal{F}_{K}$ with value in its generic point which induces a geometric point $\eta$ of $X_{K}$ via the natural (scheme theoretic) morphism $\mathcal{F}_{K} \rightarrow X_{K}$ (cf. Notations) and determines an algebraic closure $\bar{K}$ of $K$. Let $S \subset \mathcal{F}_{K}$ be a (possibly empty) finite set of closed points. We have an exact sequence of arithmetic fundamental groups

$$
1 \rightarrow \pi_{1}\left(\mathcal{F}_{K} \backslash S, \eta\right)^{\text {geo }} \rightarrow \pi_{1}\left(\mathcal{F}_{K} \backslash S, \eta\right) \rightarrow \operatorname{Gal}(\bar{K} / K) \rightarrow 1
$$

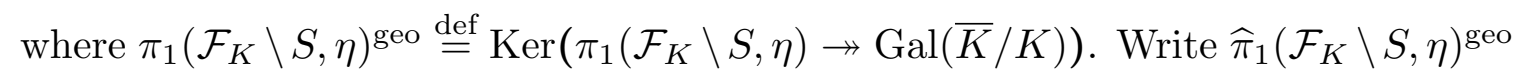
for the quotient of $\pi_{1}\left(\mathcal{F}_{K} \backslash S, \eta\right)^{\text {geo }}$ defined in 1.3.1. Thus, $\widehat{\pi}_{1}\left(\mathcal{F}_{K} \backslash S, \eta\right)^{\text {geo }}$ classifies finite (geometric) coverings $\mathcal{Y} \rightarrow \mathcal{F}$ (which we assume without loss of generality defined over $K$ ) with $\mathcal{Y}$ normal and geometrically connected, which are étale above 
$\mathcal{F}_{K} \backslash S$ and above the generic points of $\mathcal{F}_{k}$. Write $U_{K} \stackrel{\text { def }}{=} X_{K} \backslash\left(S \cup\left\{\infty_{i}\right\}_{i=1}^{n}\right)$ which is an affine curve and $\widehat{\pi}_{1}\left(U_{K} ;\left\{\infty_{i}\right\}_{i=1}^{n}, \eta\right)^{\text {geo }} \stackrel{\text { def }}{=} \widehat{\pi}_{1}\left(X_{K} \backslash\left(S \cup\left\{\infty_{i}\right\}_{i=1}^{n}\right) ;\left\{\infty_{i}\right\}_{i=1}^{n}, \eta\right)^{\text {geo }}$ for the quotient of $\pi_{1}\left(U_{K}, \eta\right)^{\text {geo }}$ defined in 1.3.1. Thus, $\widehat{\pi}_{1}\left(U_{K} ;\left\{\infty_{i}\right\}_{i=1}^{n}, \eta\right)^{\text {geo }}$ classifies finite (geometric) coverings $Y \rightarrow X$ (which we assume without loss of generality defined over $K$ ) with $Y$ normal and geometrically connected, which are étale above $X_{K} \backslash\left(S \cup\left\{\infty_{i}\right\}_{i=1}^{n}\right)$, are possibly ramified above the points $\left\{\infty_{i}\right\}_{i=1}^{n}$ with ramification indices prime-to- $p$, and are étale above the generic points of $X_{k}$. One of our main results is the following.

Theorem 3.2. The (scheme) morphism $\mathcal{F}_{K} \rightarrow X_{K}$ induces a continuous homomorphism $\widehat{\pi}_{1}\left(\mathcal{F}_{K} \backslash S, \eta\right)^{\text {geo }} \rightarrow \widehat{\pi}_{1}\left(U_{K} ;\left\{\infty_{i}\right\}_{i=1}^{n}, \eta\right)^{\text {geo }} \quad\left(\right.$ resp. $\widehat{\pi}_{1}\left(\mathcal{F}_{K} \backslash S, \eta\right)^{\text {geo }, p} \rightarrow$ $\left.\widehat{\pi}_{1}\left(U_{K} ;\left\{\infty_{i}\right\}_{i=1}^{n}, \eta\right)^{\text {geo }, p}\right)$ which makes $\widehat{\pi}_{1}\left(\mathcal{F}_{K} \backslash S, \eta\right)^{\text {geo }}\left(\right.$ resp. $\left.\widehat{\pi}_{1}\left(\mathcal{F}_{K} \backslash S, \eta\right)^{\text {geo }, p}\right)$ into a semi-direct factor of $\widehat{\pi}_{1}\left(U_{K} ;\left\{\infty_{i}\right\}_{i=1}^{n}, \eta\right)^{\text {geo }}\left(\right.$ resp. $\left.\widehat{\pi}_{1}\left(U_{K} ;\left\{\infty_{i}\right\}_{i=1}^{n}, \eta\right)^{\text {geo, } p}\right)$.

Proof. We prove the first assertion by showing the criterion in Lemma 1.1.5 is satisfied. Let $\widehat{\pi}_{1}\left(\mathcal{F}_{K} \backslash S, \eta\right)^{\text {geo }} \rightarrow G$ be a finite quotient (which we can assume without loss of generality) corresponding to a finite Galois covering $f: \mathcal{Y} \rightarrow \mathcal{F}$ with group $G$, with $\mathcal{Y}$ normal and geometrically connected, which is étale above $\mathcal{F}_{K} \backslash S$ and above the generic points of $\mathcal{F}_{k}$. We will show the existence of a surjective homomorphism $\widehat{\pi}_{1}\left(U_{K} ;\left\{\infty_{i}\right\}_{i=1}^{n}, \eta\right)^{\text {geo }} \rightarrow G$ whose composite with $\widehat{\pi}_{1}\left(\mathcal{F}_{K} \backslash S, \eta\right)^{\text {geo }} \rightarrow$ $\widehat{\pi}_{1}\left(U_{K} ;\left\{\infty_{i}\right\}_{i=1}^{n}, \eta\right)^{\text {geo }}$ is the above homomorphism. For $1 \leq i \leq n$, let $f_{i}: \mathcal{Y}_{i}=$ $\cup_{j=1}^{n_{i}} \mathcal{Y}_{i, j} \rightarrow \mathcal{X}_{i}$ be the pull-back of $f$ to $\mathcal{X}_{i}$ via the natural morphism $\mathcal{X}_{i} \rightarrow \mathcal{F}$; $\left\{\mathcal{Y}_{i, j}\right\}_{j=1}^{n_{i}}$ are the connected components of $\mathcal{Y}_{i}$ and the morphism $f_{i, j}: \mathcal{Y}_{i, j} \rightarrow \mathcal{X}_{i}$ induced by $f_{i}$ is Galois with group $G_{j}$ a subgroup of $G$. Thus, $G_{j}$ is a quotient of $\widehat{\pi}_{1}\left(\mathcal{X}_{i}, \eta_{i}\right)\left(\eta_{i}\right.$ is a suitable base point of $\left.\mathcal{X}_{i}\right)$. Fix $1 \leq j_{0} \leq n_{j}$, then $f_{i} \stackrel{\sim}{\rightarrow} \operatorname{Ind}_{G_{j_{0}}}^{G} f_{i, j_{0}}$ is an induced cover (cf. [Raynaud], 4.1). By Proposition 2.5 there exists (after possibly a finite extension of $K$ ) a finite Galois covering $\tilde{f}_{i, j_{0}}: Y_{i, j_{0}} \rightarrow D_{i}$ with group $G_{j_{0}}$, where $Y_{i, j_{0}}$ is normal and geometrically connected, whose pull-back to $\mathcal{X}_{i}$ via the natural morphism $\mathcal{X}_{i} \rightarrow D_{i}$ is isomorphic to $f_{i, j_{0}}$. Further, the morphism $\tilde{f}_{i, j_{0}}$, is ramified above $D_{i, K}$ possibly only above $\infty_{i}$ with ramification index prime-to- $p$, and $\tilde{f}_{i, j_{0}}$ is étale above the generic point of $D_{i, k}$. Let $\tilde{f}_{i}: Y_{i} \stackrel{\text { def }}{=} \operatorname{Ind}_{G_{j_{0}}}^{G} Y_{i, j_{0}} \rightarrow D_{i}$ be the induced cover (cf. loc. cit.), for $1 \leq i \leq n$. By Proposition 1.2.3 one can patch the covering $f$ with the coverings $\left\{\tilde{f}_{i}\right\}_{i=1}^{n}$ to construct a finite Galois covering $\tilde{f}: Y \rightarrow X$ between formal $R$-curves with group $G, Y$ is normal and geometrically connected (since $\mathcal{Y}_{K}$ is), which gives rise (via the formal GAGA functor) to a surjective homomorphism $\widehat{\pi}_{1}\left(U_{K} ;\left\{\infty_{i}\right\}_{i=1}^{n}, \eta\right)^{\text {geo }} \rightarrow G$ as required. Moreover, one verifies easily that the above construction can be performed in a functorial way with respect to the various quotients of $\widehat{\pi}_{1}\left(\mathcal{F}_{K} \backslash S, \eta\right)^{\text {geo }}$ (in the sense of lemma 1.1.5) using Proposition 2.5, so that one deduces the existence of a continuous homomorphism $\widehat{\pi}_{1}\left(U_{K} ;\left\{\infty_{i}\right\}_{i=1}^{n}, \eta\right)^{\text {geo }} \rightarrow \widehat{\pi}_{1}\left(\mathcal{F}_{K} \backslash S, \eta\right)^{\text {geo }}$ which is right inverse to $\widehat{\pi}_{1}\left(\mathcal{F}_{K} \backslash S, \eta\right)^{\text {geo }} \rightarrow \widehat{\pi}_{1}\left(U_{K} ;\left\{\infty_{i}\right\}_{i=1}^{n}, \eta\right)^{\text {geo }}$. The proof of the second assertion is entirely similar using similar arguments.

Proposition 3.3. The (scheme) morphism $\mathcal{F}_{K} \rightarrow X_{K}$ induces a continuous homomorphism $\widehat{\pi}_{1}\left(\mathcal{F}_{K} \backslash S, \eta\right)^{\text {geo }, p^{\prime}} \rightarrow \widehat{\pi}_{1}\left(U_{K} ;\left\{\infty_{i}\right\}_{i=1}^{n}, \eta\right)^{\text {geo }, p^{\prime}}$ which makes $\widehat{\pi}_{1}\left(\mathcal{F}_{K} \backslash\right.$ $S, \eta)^{\text {geo, } p^{\prime}}$ into a semi-direct factor of $\widehat{\pi}_{1}\left(U_{K} ;\left\{\infty_{i}\right\}_{i=1}^{n}, \eta\right)^{\text {geo }, p^{\prime}}$.

Proof. The proof follows by using similar arguments to the ones used in the proof of Theorem 3.2. More precisely, with the notations in the proof of Theorem 3.2 
the morphism $\mathcal{Y}_{i, j} \rightarrow \mathcal{X}_{i}$ in this case is Galois with group $\mu_{n}$, where $n$ is an integer prime-to- $p$, and extends (uniquely, possibly after a finite extension of $K$ ) to a cyclic Galois covering $Y_{i, j} \rightarrow D_{i}$ of degree $n$ ramified only above $\infty_{i}$ (cf. Lemma 2.1 and Lemma 2.4).

In [Saïdi1] we defined the genus $g_{x}$ of the closed point $x$ of $X$, whose definition depends only on the local (étale) structure of $X_{k}$ at $x$, and which equals the genus of the proper, connected, and smooth $K$-curve $X_{K}$ constructed above (cf. loc. cit. Lemma 3.3.1 and the discussion before it). (The genus $g_{x}$ of $x$ is also called the genus of the formal fibre $\mathcal{F}_{K}$.)

Theorem 3.4. Let $S(\bar{K})=\left\{y_{1}, \ldots, y_{m}\right\}$ of cardinality $m \geq 0$. Then the continuous homomorphism $\pi_{1}\left(\mathcal{F}_{K} \backslash S, \eta\right)^{\text {geo }, p^{\prime}} \rightarrow \pi_{1}\left(X_{K} \backslash\left(S \cup\left\{\infty_{i}\right\}_{i=1}^{n}\right), \eta\right)^{\text {geo, } p^{\prime}} \quad(c f$. Proposition 3.3) is an isomorphism. In particular, $\pi_{1}\left(\mathcal{F}_{K} \backslash S, \eta\right)^{\text {geo, } p^{\prime}}$ is (pro)prime-to- $p$ free of rank $2 g_{x}+n+m-1$ and can be generated by $2 g_{x}+n+m$ generators $\left\{a_{1}, \ldots, a_{g}, b_{1}, \ldots, b_{g}, \sigma_{1}, \ldots, \sigma_{n}, \tau_{1}, \ldots, \tau_{m}\right\}$ subject to the unique relation $\prod_{i=1}^{g}\left[a_{i}, b_{i}\right] \prod_{j=1}^{n} \sigma_{j} \prod_{t=1}^{m} \tau_{t}=1$, where $\sigma_{j}$ (resp $\left.\tau_{t}\right)$ is a generator of inertia at $\infty_{i}\left(\right.$ resp. $\left.y_{t}\right)$.

Proof. The homomorphism $\pi_{1}\left(\mathcal{F}_{K} \backslash S, \eta\right)^{\text {geo, } p^{\prime}} \rightarrow \pi_{1}\left(X_{K} \backslash\left(\left\{\infty_{i}\right\}_{i=1}^{n} \cup S\right), \eta\right)^{\text {geo }, p^{\prime}}$ is injective as follows from Proposition 3.3 (note that $\widehat{\pi}_{1}=\pi_{1}$ in this case). We show it is surjective. To this end it suffices to show that given a finite Galois covering $f: Y \rightarrow X$ with group $G$ of cardinality prime-to- $p$, with $Y$ normal and geometrically connected, which is étale above $X_{K} \backslash\left(S \cup\left\{\infty_{i}\right\}_{i=1}^{n}\right)$, and $\tilde{f}: \mathcal{Y}_{K} \rightarrow \mathcal{F}_{K}$ its restriction to $\mathcal{F}_{K}$, then $\mathcal{Y}_{K}$ is geometrically connected. Equivalently, we need to show (possibly after passing to a finite extension of $K$ ) that $f^{-1}(x)$ consists of a single closed point. (The set of connected components of $\mathcal{Y}_{K}$ is in one-to-one correspondence with the set $f^{-1}(x)$.) We can assume, without loss of generality, that $Y_{k}$ is reduced (cf. Lemme d'Abhyankar, [Grothendieck], Exposé X, Lemme 3.6). Let $y \in f^{-1}(x)$ and $D_{y} \subset G$ its decomposition group. Let $Y_{i}$ be an irreducible component of $Y_{k}$ above $P_{i}$ passing through $y, \widetilde{Y}_{i} \rightarrow Y_{i}$ the morphism of normalisation, and $\widetilde{Y}_{i} \rightarrow \widetilde{P}_{i}$ the natural morphism which is Galois with group $D_{Y_{i}} \subset G$ the decomposition group of $Y_{i}$. The morphism $\widetilde{Y}_{i} \rightarrow \widetilde{P}_{i}$ is étale outside $\left\{x_{i}, \infty_{i}\right\}$ by Zariski's purity Theorem. Hence $D_{Y_{i}}=\mu_{n}$ is cyclic of order $n$, for some integer $n$ prime-to- $p$, and the above morphism $\widetilde{Y}_{i} \rightarrow \widetilde{P}_{i}$ is totally ramified above $\infty_{i}$ and $x_{i}$ as follows from the structure of $\pi_{1}\left(\mathbb{P}_{\bar{k}}^{1} \backslash\{0, \infty\}\right)^{p^{\prime}}$. In particular, $D_{Y_{i}} \subset D_{y}$. Moreover, $Y_{k}$ is regular outside $f^{-1}(x)$ (cf. [Raynaud], Lemma 6.3.2). We can associate a graph $\Gamma$ to $Y_{k}$ whose vertices are the irreducible components of $Y_{k}$ and edges are the closed points of $Y_{k}$ above $x$, two vertices $Y_{i}$ and $Y_{i^{\prime}}$ passing by a closed point $y$ above $x$ are linked by the edge $y$. Assume that $f^{-1}(x)$ has cardinality $>1$ and let $\left\{y, y^{\prime}\right\} \subseteq f^{-1}(x)$ be two distinct points. Then no irreducible component of $Y_{k}$ passes through both $y$ and $y^{\prime}$ (cf. the above fact that $\widetilde{Y}_{i} \rightarrow \widetilde{P}_{i}$ is totally ramified above $x_{i}$ ). More precisely, if $Y_{i}$ is an irreducible component of $Y_{k}$ then $Y_{i}$ passes through a unique point $y$ of $Y_{k}$ above $x$. From this (and the above facts) it follows easily that the connected components of $\Gamma$ are in one-to-one correspondence with the elements of $f^{-1}(x)$ and $\Gamma$ is disconnected which contradicts the fact that $Y_{k}$ is connected. Thus, $f^{-1}(x)$ has cardinality 1 necessarily as required. The last assertion follows form the well-known structure of $\pi_{1}\left(X_{K} \backslash\left(S \cup\left\{\infty_{i}\right\}_{i=1}^{n}\right), \eta\right)^{\text {geo, } p^{\prime}}$ (cf. [Grothendieck], Exposé XIII, Corollaire 2.12). 
Examples 3.5. Suppose $K$ is of mixed characteristics with $\operatorname{char}(k)=p>0$. Let $\mathcal{F}=\operatorname{Spf} R[[T]]$ (resp. $\mathcal{F}=\operatorname{Spf} R\left[\left[T_{1}, T_{2}\right]\right] /\left(T_{1} T_{2}-\pi^{e}\right)$ ) be the formal open unit disc (resp. formal open annulus of thickness $e \geq 1$ ) and $S=\left\{y_{1}, \ldots, y_{m}\right\} \subset \mathcal{F}(K)$ a set of $m \geq 0$ distinct $K$-rational points (in the second case $e>1$ necessarily if $m \neq 0$ ). In this case $\mathcal{F}$ has a compactification $X=\mathbb{P}_{R}^{1}$ the $R$-projective line with parameter $T$ and $\mathcal{F}$ is the formal germ at $T=0$ (resp. a compactification $X$ which is a formal model of the projective line $\mathbb{P}_{K}^{1}$ consisting of two formal closed unit discs $D_{1}$ and $D_{2}$ centred at $\infty_{1}$ and $\infty_{2}$; respectively, which are patched with $\mathcal{F}$ along its two boundaries. The special fibre $X_{k}$ consists of two projective lines which intersect at the double point $x$ and $\mathcal{F}$ is the formal germ at $x)$. The results of $\S 3$ in this case read as follows. First, the homomorphism $\widehat{\pi}_{1}\left(\mathcal{F}_{K} \backslash S, \eta\right)^{\text {geo }} \rightarrow \widehat{\pi}_{1}\left(\mathbb{P}_{K}^{1} \backslash(T \cup\{\infty\}) ;\{\infty\}, \eta\right)^{\text {geo }}\left(\right.$ resp. $\widehat{\pi}_{1}\left(\mathcal{F}_{K} \backslash S, \eta\right)^{\text {geo }} \rightarrow \widehat{\pi}_{1}\left(\mathbb{P}_{K}^{1} \backslash\right.$ $\left.\left.\left(T \cup\left\{\infty_{1}, \infty_{2}\right\}\right) ;\left\{\infty_{1}, \infty_{2}\right\}, \eta\right)^{\text {geo }}\right)$ makes $\pi_{1}\left(\mathcal{F}_{K} \backslash S, \eta\right)^{\text {geo }}$ into a semi-direct factor of $\widehat{\pi}_{1}\left(\mathbb{P}_{K}^{1} \backslash(T \cup\{\infty\}) ;\{\infty\}, \eta\right)^{\text {geo }}\left(\right.$ resp. $\left.\widehat{\pi}_{1}\left(\mathbb{P}_{K}^{1} \backslash\left(T \cup\left\{\infty_{1}, \infty_{2}\right\}\right) ;\left\{\infty_{1}, \infty_{2}\right\}, \eta\right)^{\text {geo }}\right)$ and the maximal prime-to- $p$ quotient $\pi_{1}\left(\mathcal{F}_{K} \backslash S, \eta\right)^{\text {geo, } p^{\prime}}$ is free of rank $m$ (resp. $m+1)$.

\section{References.}

[Bosch] S. Bosch, Eine bemerkenswerte Eigenshaft des formellen Fasern affinoider Räume, Math. Ann. 229 (1977), 25-45.

[Bosch-Lütkebohmert] S. Bosch and W. Lütkebohmert, Stable reduction and uniformisation of abelian varieties I, Math. Ann. 270 (1985), 349-379.

[Bourbaki] N. Bourbaki, Algèbre Commutative, Chapitre 9, Masson, 1983.

[Garuti] M. Garuti, Prolongements de revêtements galoisiens en géométrie rigide, Compositio Mathematica, 104 (1996), no 3, 305-331.

[Grothendieck] A. Grothendieck, Revêtements étales et groupe fondamental, Lecture Notes in Math. 224, Springer, Heidelberg, 1971.

[Harbater] D. Harbater, Galois groups and fundamental groups, 313-424, Math. Sci. Res. Inst. Publ., 41, Cambridge Univ. Press, Cambridge, 2003.

[Raynaud] M. Raynaud, Revêtements de la droite affine en caractéristique $p>0$ et conjecture d'Abhyankar, Invent. Math. 116 (1994), no 1-3, 425-462.

[Ribes-Zalesskii] L. Ribes and P. Zalesskii, Profinite groups, Ergebnisse der Mathematik und ihrer Grenzgebiete. Folge 3. A series of Modern Survey in Mathematics 40. Springer-Verlag, Berlin 2000.

[Pries] R. Pries, Construction of covers with formal and rigid geometry, in: J. -B. Bost, F. Loeser, M. Raynaud (Eds.), Courbes semi-stables et groupe fondamental en géométrie algébrique, Progr. Math., Vol. 187, 2000.

[Saïdi] M. Saïdi, Étale fundamental groups of affinoid $p$-adic curves, Journal of algebraic geometry, 27 (2018), 727-749.

[Saïdi1] M. Saïdi, Wild ramification and a vanishing cycles formula, J. Algebra 273 (2004), no. 1, 108-128.

[Serre] J-P. Serre, Cohomologie Galoisienne, Lecture Notes in Math., 5, Springer Verlag, Berlin, 1994.

[Serre1] J-P. Serre, Construction de revêtements étale de la droite affine en caractéristique $p>0$, C. R. Acad. Sci. Paris 311 (1990), 341-346.

Mohamed Saïdi

College of Engineering, Physics and Mathematical Sciences 
University of Exeter

Harrison Building

North Park Road

EXETER EX4 4QF

United Kingdom

M.Saidi@exeter.ac.uk 